Dossiê 8

Práticas interacionais na construção

\title{
UM OLHAR SOBRE FORMAS E COMPOSIÇÕES NO DESIGN DE INTERIORES SOB A ÓTICA DA ARTE 1
}

\section{A point of view about forms and compositions in interior design through the optics of art}

\section{Una mirada a las formas y composiciones en diseño de interiores mediante de la óptica del arte}

\author{
Ana Beatriz Alonso de Oliveira ${ }^{2}$ \\ Luciene Contiero Felipe ${ }^{3}$ \\ Marcus Vinicius Pereira ${ }^{4}$
}

\footnotetext{
${ }^{1}$ Este artigo é o resultado da monografia "Um olhar sobre formas e composições no Design de Interiores através da ótica da arte" realizado pelas alunas Ana Beatriz Alonso de Oliveira e Luciene Contiero Felipe como trabalho final do curso de Especialização em Projetos de Interiores, sob orientação do Prof. Ms. Marcus Vinicius Pereira.

${ }^{2}$ Arquiteta, Especialista em Design de Interiores e Professora Assistente de História da Arte na Escola da Cidade. Pós-graduação latu senso em Arquitetura, Educação e Sociedade. Lattes: http://lattes.cnpq.br/5058642606240927. Orcid: https://orcid.org/0000-0003-1684-0234. E-mail: arq.anabeatrizalonso@gmail.com.

${ }^{3}$ Designer de Interiores e especialista em Projeto de Interiores. Profissional autônoma com atuação em design de interiores residencial, integra conceitos da arte no desenvolvimento de seus projetos. Lattes: http://lattes.cnpq.br/8683491220190733. Orcid: https://orcid.org/0000-0002-2696-2203. Email: luciene.toysvilla@gmail.com

${ }^{4}$ Arquiteto, Especialista em Design de Interiores, Mestre e Doutorando em Design. Professor Universitário da Universidade Anhembi Morumbi nos cursos de Arquitetura e Urbanismo e Design de
} 


\title{
RESUMO
}

Este artigo é o desdobramento de uma monografia de pós-graduação que analisou o livro "Universos da Arte", de Fayga Ostrower (2013), e sua relação com o design de interiores. Utilizou-se como base o experimento apresentado na obra em questão, em que a autora aborda as aulas que ministrou para operários de fábrica, sobre conceitos de composição e forma, com o objetivo de auxiliá-los a compreender obras de arte. No primeiro momento da pesquisa, contextualiza-se como foi feito esse experimento e quais são os conceitos abordados. Em seguida, demonstra-se sua aplicabilidade no design de interiores, por meio da análise da correlação dos projetos da arquiteta e designer Patricia Urquiola e de sua aplicação sob a ótica central do tema.

Palavras-chaves: Fayga Ostrower; gestalt; design de interiores; Patricia Urquiola.

\begin{abstract}
This article has the objective to communicate a postgraduate monograph, which is an analysis of the book Art Universes by Fayga Ostrower and it's relationship with interior design. In order to do so, the experiment shown in the book was used as the basis, in which the author discusses the classes she taught to factory workers some concepts of composition and form in order to help them understand artworks. At first, research contextualizes how this experiment was made and what are these concepts about, to then demonstrate its applicability in interior design. This can be noticed through the analysis made between the correlation of the architect and designer Patricia Urquiola's projects with the application from the central point of view of the theme.
\end{abstract}

Keywords: Fayga Ostrower, gestalt, interior design, Patricia Urquiola.

\section{Resumen}

Este artículo tiene el objetivo de comunicar una monografía de posgrado, que es un análisis del libro Art Universes de Fayga Ostrower y su relación con el diseño de interiores. Para hacerlo, se utilizó como base el experimento que se muestra en el libro, donde la autora discute las clases que enseñó a los trabajadores de una fábrica algunos conceptos de composición y forma para ayudarlos a comprender las obras de arte. Al principio, la investigación contextualiza cómo se realizó este experimento y de qué se tratan estos conceptos, para luego demostrar su aplicabilidad en el diseño de interiores. Esto se puede notar mediante del análisis realizado entre la correlación de los proyectos del arquitecto y diseñador Patricia Urquiola con la aplicación desde el punto de vista central del tema.

Palavras clave: Fayga Ostrower; gestalt; diseño de interiores; Patricia Urquiola.

Interiores e na Pós-graduação latu senso em Projeto de Interiores. Suas pesquisas fazem conexões entre as áreas da arquitetura, design de moda e filosofia, abordando conceitos como efemeridade, deslocamento e processos de criação. Lattes: http://lattes.cnpq.br/5597420319745243. Orcid: https://orcid.org/0000-0001-5114-0562. E-mail: mvpereira@anhembi.br 


\section{INTRODUÇÃO}

A arte está diretamente relacionada a diversos campos de atuação, tais como cenografia, música, dança, fotografia, moda etc., que dialogam e contribuem para as obras de artistas de variadas áreas.

Este artigo apresenta a síntese de um trabalho de monografia de pósgraduação, cuja pesquisa possui caráter exploratório, com dados levantados a partir do livro "Universos da Arte", de Fayga Ostrower". A obra trata de um experimento desenvolvido pela autora com uma turma de operários, por meio do qual ela ministra aulas com o intuito de contribuir para que os alunos possam, de fato, compreender obras de arte.

Ao entrar no universo da arte, alguns conceitos são estabelecidos por meio da análise de espaço, forma, elementos visuais e composição, apresentados com o respaldo de elementos iconográficos do livro.

A partir desses tópicos, foi construída a narrativa que apresenta uma análise aprofundada sobre eles, com o intuito de mostrar a aplicação dos conceitos e como estes podem funcionar como uma diretriz, ao se conceber um novo espaço.

Nesse contexto, os projetos da arquiteta e designer de interiores Patrícia Urquiola revelam as aplicações e o resultado obtido através dessa ligação entre a ótica da arte e o design.

\section{DESENVOLVIMENTO}

\subsection{Conceitos e análise de questões das formas e composições}

Ao apresentar os conceitos de composição e forma para a turma de operários, estes foram conduzidos a compreender a percepção de espaço nas obras de arte estudadas.

\footnotetext{
${ }^{5}$ Fayga Ostrower nasceu em 1920, na cidade de Lodz, Polônia, e chegou ao Rio de Janeiro em 1934. Atuava como gravadora, pintora, desenhista, ilustradora, teórica da arte e professora. Entre os anos de 1954 e 1970, desenvolveu atividades docentes no MAM - Museu de Arte Moderna, no Rio. Durante esse período, desenvolveu também cursos para operários e centros comunitários, visando à divulgação da arte (Fonte: FAIGA OSTROWER. Disponível em: <https://faygaostrower.org.br/>. Acesso em: 27 maio 2020).
} 
Com um método de interação com os alunos, Ostrower colocou em prática experimentos que estimulavam a sensibilidade ao analisar a arte, além de determinadas formas expressivas que surgem em relação a culturas distintas. Também apresentou alguns conceitos para aguçar essas percepções, como é o caso da gestalt 6 .

A teoria da gestalt provém da psicologia de experimentação e sugere uma resposta ao porquê de algumas formas agradarem mais do que outras. Trata-se do resultado de uma integração de partes em oposição à soma do "todo" (GOMES FILHO, 2009). Em uma explicação simplista, pode-se fazer a seguinte analogia: quando se observa um armário, deve-se reparar no todo e não em cada parte deste, como dobradiças, prateleiras, espelho etc.

No âmbito da composição de um espaço, as formas podem demonstrar emoção, tranquilidade, movimentos bruscos, enfim, conteúdos expressivos, que podem coexistir no mesmo espaço e ser percebidos tanto na arte como na vida. Essas formas são expressas e interpretadas conforme a visão pessoal de cada indivíduo, e refletem sua experiência de vida, valores, entre outros.

Segundo Ostrower (2013, p.60), "em toda obra de arte, a forma incorpora o conteúdo de tal modo que se torna uma só identidade"; assim, a cada modificação da forma, modifica-se também o conteúdo.

Ainda conforme a autora, pode-se observar como uma única forma modifica todo o espaço em que está inserida - a dinâmica do espaço, seu equilíbrio, movimento -, levando a várias possibilidades. Contornos e limites determinam a forma e, quando se percebem tais limites, percebe-se, também, sua estrutura, o que implica dizer que a forma sempre estará interligada à organização, ordenação e estrutura.

Os elementos visuais apresentam valores diferentes, tanto em termos de estrutura do espaço como de expressividade. Isso pode ser demonstrado, por exemplo, por meio de seu posicionamento. Se um determinado elemento visual estiver

\footnotetext{
${ }^{6} \mathrm{~A}$ tradução de gestalt, em inglês, espanhol e português, é geralmente relacionada a estrutura, figura e forma, e se trata de uma teoria que sugere uma resposta ao porquê de certas formas agradarem mais, e outras menos, as pessoas (GOMES FILHO, 2009, p.18).
} 
posicionado no alto ou na parte inferior do plano, terá expressividades e estruturas de espaço diferentes.

Figura 1 - Desenho representativo do quadro-negro.
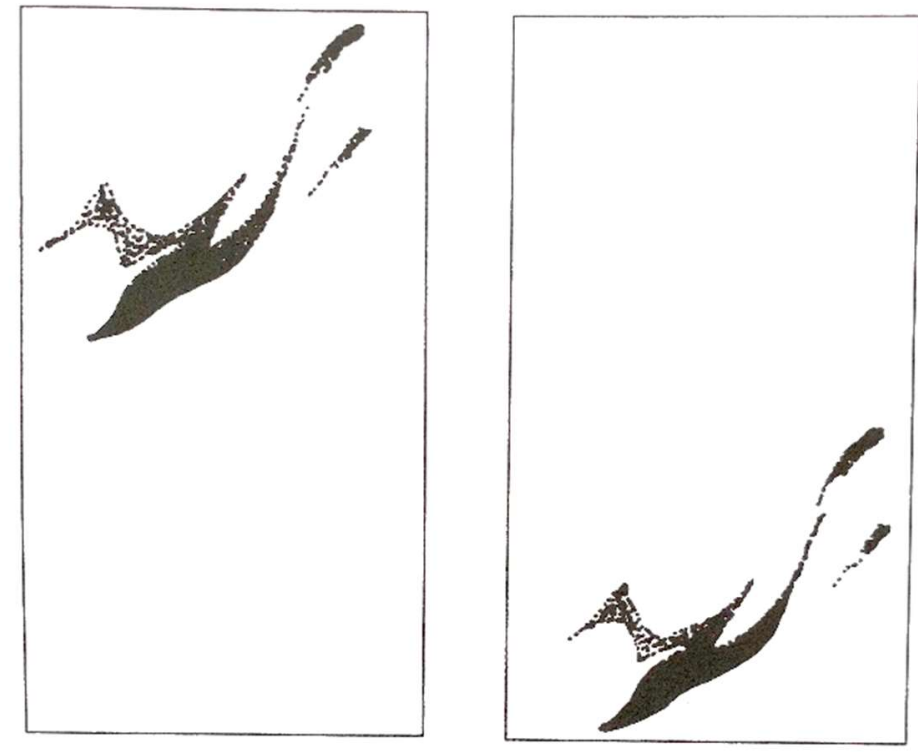

Fonte: Ostrower (2013. p. 73).

Na figura 1, pode-se observar a mesma forma colocada no alto e na parte baixa do plano. Quando posicionada acima, expressa leveza e movimento, e quando situada na parte de baixo, observa-se maior peso e menor movimento. Outro ponto interessante é perceber a importância do elemento visual no canto inferior direito de uma composição, pois normalmente leva o observador a "sair" desta área, em que parece haver uma maior concentração de energia, tornando-a, segundo Ostrower (2013), uma área de ação ou de conclusão, mais densa.

É curiosa a tendência do ser humano em perceber o movimento de orientação da lateral esquerda para a direita, quando se olha para uma obra, pois não se "entra" nela pelo centro, apesar de a visão ser frontal e paralela ao plano observado.

Sobre composição, o artista pode utilizar semelhanças, contrastes ou ambos. Com semelhanças, ele conseguirá sequências rítmicas, e com contrastes, tensões espaciais, podendo alcançar imagens com características líricas ou dramáticas (OSTROWER, 2013), que podem apresentar diversas possibilidades de variantes formais para cada elemento, seja com superfícies (abertas e fechadas), volumes (cheios e vazios), luz (claro e escuro) ou cor. 
Certos tipos de contrastes e semelhanças formais nos levam a identificar os elementos com que o artista trabalha. E, a partir dos elementos, podemos compreender o caráter do espaço que foi ordenado através desses elementos e, sobretudo, o caráter expressivo da ordenação. É o que nos dará os significados de uma obra (OSTROWER, 2013, p. 219).

Percebe-se que, em uma análise da obra, os contrastes guiam, fazendo o observador parar ou dar continuidade ao percorrer o quadro. Com isso, transmitemse conteúdos emotivos, dramáticos ou contemplativos, gera-se um ritmo ou tensão espacial e criam-se dois aspectos fundamentais do movimento: espaço e tempo (OSTROWER, 2013).

Essa imersão no tocante aos conceitos e estratégias aplicados em obra de arte, permite que se transmute sua aplicação para o design de interiores.

\subsection{Aplicação e influência no design de interiores}

Ao iniciar um projeto, deve-se pensar em seu todo. Seguindo os mesmos princípios da gestalt, faz-se necessário valorizar as partes, mas sempre tendo em mente sua totalidade, a unidade do espaço.

Segundo Gibbs (2010), a primeira etapa de qualquer projeto consiste na reunião e análise da informação, constituindo-se, assim, a base de trabalho que possibilitará ao designer de interiores desenvolver suas ideias. Essa etapa define-se como delimitação inicial, fase na qual serão definidos os fluxos do projeto, indicandose como será a circulação, até que ponto ela irá levar, espaços de entrada e saída e como os espaços conversam.

A segunda etapa é o desenvolvimento criativo. Este, apesar de ser, em grande parte, intuitivo, é norteado por certos princípios e normas fundamentais de design, estabelecidos ao longo do tempo. Embora possam mudar conforme a época, sua finalidade sempre foi a mesma: criar espaços equilibrados e harmoniosos de acordo com funções e necessidades. Cabe ressaltar que o designer de interiores, para obter esse resultado, deve compreender e considerar os seguintes aspectos: dimensionamento humano, escala e proporção e princípios de ordem (GIBBS, 2010).

A delimitação inicial servirá de guia até o final do projeto, com o intuito de auxiliar a distribuir o programa e até mesmo moldar e induzir o desenho do projeto. Para Grimley, "o designer de interiores deve se envolver com o processo de criar uma 
coreografia de espaços, de modo que o projeto reflita uma única abordagem de design" (2017, p. 84).

A escolha temática vincula-se a aspectos afetivos, dos quais o artista, na maioria das vezes, nem se dá conta. É uma questão de afinidade pessoal, uma seletividade que não se restringe ao tema, mas abrange, também, o enfoque formal do artista e envolve, de uma só vez, todos os aspectos técnicos e estilísticos do seu trabalho (OSTROWER, 2013). Isso reforça a importância de o artista ser claro na linguagem que usa e, para tanto, é preciso que, no projeto, sejam claramente reconhecíveis os elementos de sua linguagem visual, através de ritmos, contrastes ou cores. É muito comum, por exemplo, se olhar um projeto e saber de quem é, devido a essa linguagem visual marcante, que acaba se tornando uma característica única do artista.

Para Arnheim (2004, p.29), “a nossa mente organiza as inúmeras estruturas com o auxílio de dois processos cognitivos: a percepção intuitiva e a análise intelectual". Desse modo, a percepção de cada indivíduo organiza-se por meio da seletividade sobre suas necessidades físicas e psicológicas e forma um esquema de prioridades internas, que prende a atenção para certas eventualidades.

Há certas qualidades comuns a todos os designers de sucesso. Obviamente, uma condição sine qua non é possuir um grande domínio do funcionamento do ofício: o planejamento do espaço, a combinação dos materiais, a compreensão da cor. Mas todas essas qualidades são inúteis se não estiverem relacionadas a um rigoroso controle dos detalhes e à habilidade tenaz de materializar as ideias (GIBBS, 2010, p.8).

No caso do design de interiores, existem alguns processos de planejamento e organização desses elementos. Questões como: (1) as características do local e a função do interior proposto; (2) o desenvolvimento de ideias conceituais; (3) a representação das organizações espaciais (com os tipos de desenhos e diagramas usados para apresentar o projeto); (4) o detalhamento do desenvolvimento do projeto e (5) a execução da obra são alguns dos processos de concepção do projeto.

Ao projetar, sempre se pensa em uma determinada área e onde, nesta, deverão ser propostas margens, delimitando-se, ainda, onde começa e onde termina o projeto. Essas margens podem ser paredes, elementos estruturais, paisagismo etc. e causam o efeito de estabilizar o movimento visual. Podem ocorrer em duas direções: na vertical e na horizontal. 
Para o ser humano, essas direções têm um sentido bastante específico e a horizontal, especialmente, é percebida como uma posição "deitada", transmitindo uma ideia de sono, repouso, morte, calma, mas sempre associada à imobilidade e à ausência de movimento (OSTROWER, 2013).

Quanto às cores, segundo Gibbs (2010), as pessoas podem não mencionar o esquema cromático de um projeto, mas, certamente, comentarão que um determinado ambiente é muito acolhedor, cálido, convidativo, limpo, espaçoso, elegante ou intimista - impressões diretamente provocadas pelas tonalidades de cor utilizadas.

A aplicação e mistura das cores, há muito tempo, tem sido uma intensa área de estudos para cientistas, artistas e designers. A cor, apesar de explícita, pode ser, ao mesmo tempo, um tópico extremamente subjetivo, uma vez que os indivíduos têm suas cores favoritas: aquelas que lembram um lugar, remetem a um momento ou possuem características emocionais específicas. Nesse contexto, Silva aborda a relação das cores com as emoções:

\begin{abstract}
A cor se revela na visão. A emoção não está apenas contida na percepção, mas também na impulsividade, e é interpretada duplamente como paixão e ação da luz. Nessa perspectiva, existe um elo entre cor e luz, porém a cor não é apenas constituída de luz, mas um impulso originado da paixão contida no olhar que toma forma e cria a natureza. No círculo cromático feito à base de aquarela, pode-se notar como Goethe se esforça em relacionar as manchas cromáticas com as emoções humanas: razão, fantasia, entendimento e sensibilidade (GOETHE, 2013 apud SILVA, 2016, p.24).
\end{abstract}

O papel da cor no design de interiores resiste a uma explicação por meio de simples regras e ideias; ainda assim, entender as complexidades do uso da cor em um espaço é fundamental para se criar um interior bem-sucedido. Os designers de interiores devem, portanto, aprender as características das cores e saber como elas trabalham como agente ordenador e como forma de atrair a atenção (GRIMLEY, 2017).

Quando utilizadas com conhecimento e cuidado, as cores podem aumentar o peso visual das superfícies, alterar as proporções básicas de um recinto e ser um fator de relaxamento ou excitação. À medida que o design começa a explorar e entender os efeitos superficiais das cores, elas se tornam a base de uma rica paleta visual e material (GRIMLEY, 2017). 


\subsection{PATRICIA URQUIOLA}

Com o objetivo de se aplicarem os conceitos e métodos propostos na obra de Faiga Ostrower, objeto deste estudo, selecionou-se o abrangente portfólio da arquiteta espanhola Patricia Urquiola ${ }^{7}$, visando a explorar as suas principais características e fazer uma correlação dos seus trabalhos com a perspectiva do tema.

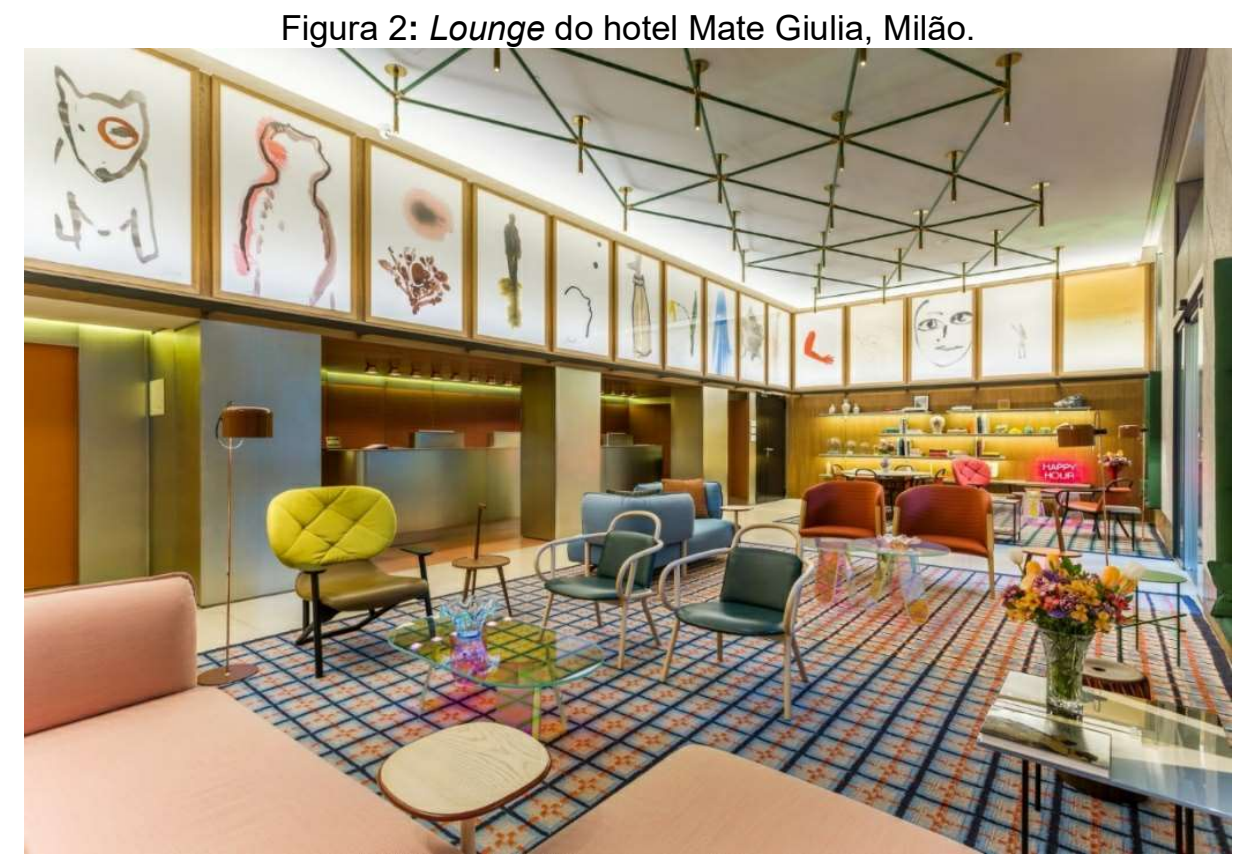

Fonte: Disponível em: <https://room-matehotels.com/en/giulia/>. Acesso em: 19 maio 2020.

A figura 2 mostra um de seus projetos, o hotel Mate Giulia ${ }^{8}$, no qual podese notar, no ambiente do loung ${ }^{9}$, que as diagonais do plano superior orientam o olhar para a contemplação das gravuras que emolduram o espaço. Logo após, o tapete convida a perceber o mobiliário. Por estarem em um plano superior, as diagonais tendem a "pesar" e até incomodar, porém ao se utilizarem linhas finas e repetidas, se estabelece uma unidade e proporciona-se delicadeza ao projeto.

\footnotetext{
${ }^{7}$ A arquiteta, radicada na Itália, é um dos grandes nomes da arquitetura e do design de mobiliário contemporâneo (Fonte: Disponível em: <https://habitusbrasil.com/patricia-urquiola-designarquitetura/>. Acesso em: 28 maio 2020.

${ }^{8}$ O hotel está localizado em Milão, na Itália, e o projeto foi feito a partir de um edifício histórico do final do século XIX. O hotel incluiu em seu programa um spa, salas de reuniões no porão, lobby, sala de café da manhã, restaurante e 85 quartos e suítes, distribuídos em seis pavimentos (Fonte: Disponível em: <www.archilovers.com/projects/176656/hotel-room-mate-giulia-milan.html\#info>. Acesso em: 28 maio 2020).

${ }^{9} \mathrm{O}$ termo em inglês lounge significa um espaço onde as pessoas podem relaxar ou esperar (Fonte: Disponivel em: <https://dictionary.cambridge.org/pt/dicionario/ingles/lounge $\geq$. Acesso em: 26 maio 2020).
} 
Figura 3: Lounge do hotel Mate Giulia 3, Milão.

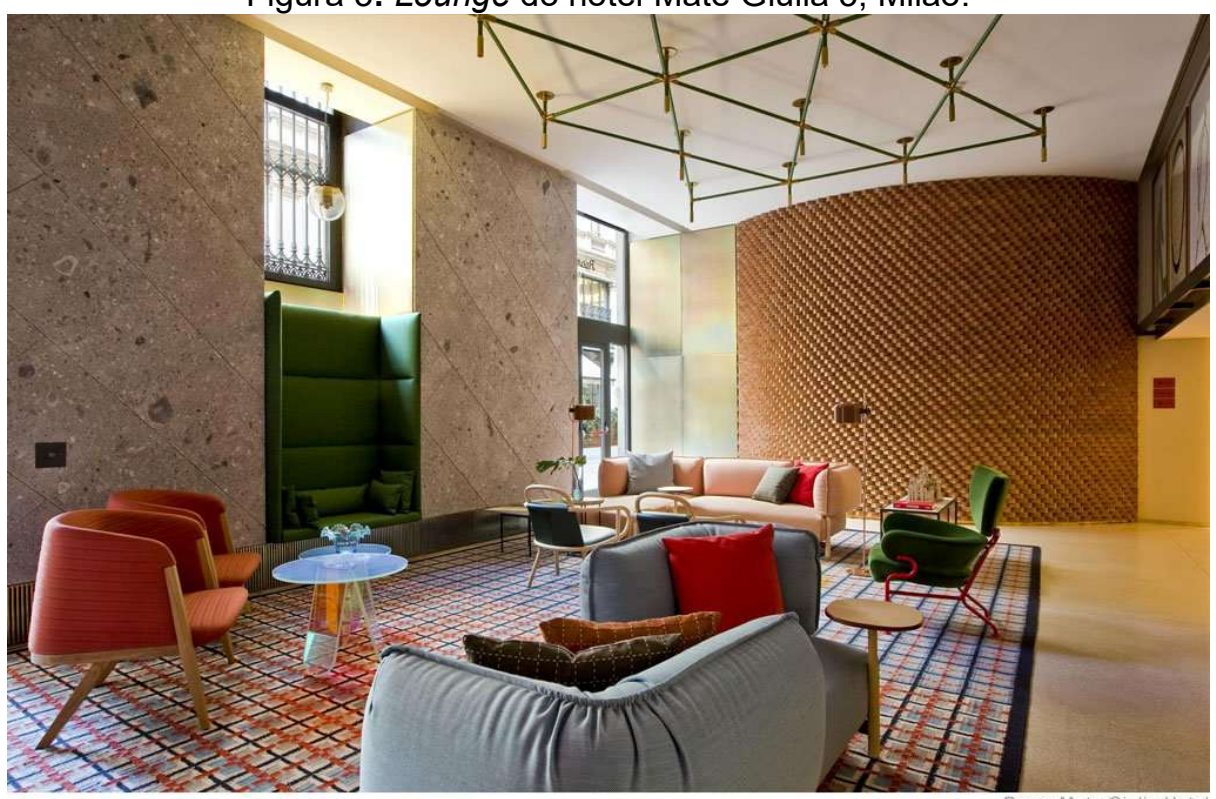

Fonte: Disponível em: <https://room-matehotels.com/en/giulia/>. Acesso em: 19 maio 2020.

Em contraponto, na figura 3, pode-se perceber que o tapete possui linhas verticais e horizontais, formando quadrados que equilibram o movimento superior, como se fossem setas, que guiam o olhar em direção ao mobiliário e delimitam o ambiente. Formas orgânicas, presentes em gravuras e também nos mobiliários, proporcionam a quebra da rigidez das formas geométricas e tornam a composição leve, alegre e harmônica.

Segundo Ferraz (2016), a percepção humana tende a direcionar maior peso visual às partes inferiores dos objetos, sendo assim, quando se fala na ordenação de movimento, os artistas têm uma predisposição a distribuí-los em diagonais, que se deslocam de cima para baixo e da esquerda para a direita. A partir disso, conclui-se que o ser humano idealiza dessa maneira o deslocamento dos objetos nos espaços.

Nota-se o equilíbrio na posição e nas cores do mobiliário, volumes maiores encontram-se em contraposição diagonal, assim como os menores e suas cores complementares posicionados em diagonal oposta. Embora possua diversas cores, texturas, movimento, volumes e formas em sua composição, o espaço transmite uma atmosfera harmônica.

É necessário definir o plano pictórico, para proporcionar harmonia entre os elementos visuais. Os volumes maiores estão dispostos em contraposição diagonal e 
os menores em diagonal oposta; destacam-se, ainda, as cores complementares e os elementos visuais, que orientam e direcionam o olhar.

Figura 4: Lounge do Hotel Mandarin, Barcelona.

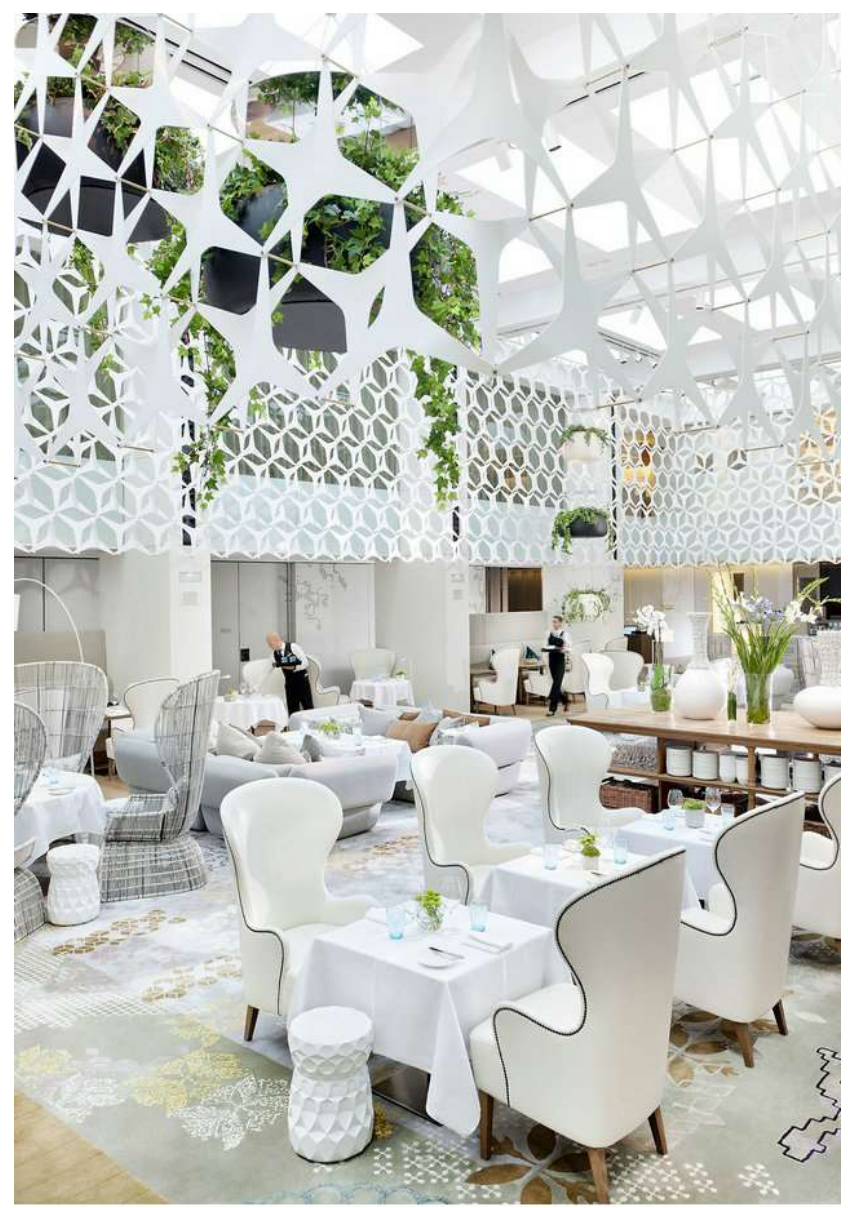

Fonte: Disponível em: <https://www.archdaily.com.br/br/01-76664/hotel-mandarin-oriental-barcelonaoab?ad_medium=gallery>. Acesso em: 19 maio 2020.

Na figura 4, pode-se ver o lounge do Hotel Mandarim ${ }^{10}$ : suas estruturas metálicas são o ponto de atração principal e, apesar do material, trazem leveza e delimitam o espaço. Com tantas informações na parte superior, a parte inferior segue com a mesma cor e poucos pontos coloridos, trazendo luminosidade e unidade ao ambiente.

O mobiliário foi utilizado de formas distintas no plano inferior, razão pela qual não se tem a impressão de um espaço visualmente poluído ou com excessos. Percebe-se como o espaço modifica-se, quando se acrescentam elementos que o

10 O hotel está localizado no coração de Barcelona, na Espanha. É um edifício do século XX, anteriormente sede do Banco Hispano-Americano e foi transformado em hotel. Patricia Urquiola renovou o seu interior (Fonte: Disponível em: <www.archdaily.com.br/br/01-76664/hotel-mandarinoriental-barcelona-oab?ad_medium=gallery>._Acesso em: 28 maio 2020). 
tornam mais decorativo ou introduzem um maior movimento visual, até chegar a um ponto de equilíbrio, sem se tornar, contudo, supérfluo em sua composição.

A partir da análise dos projetos de Urquiola, fica nítida a aplicabilidade dos conceitos apresentados, e se pode observar a importância de sua aplicação. A Gestalt, por exemplo, é fundamental, visto o desafio de haver uma grande quantidade de elementos nos projetos e, ainda assim, estabelecer uma unidade. Isso reforça a ideia de que a compreensão das formas visuais é a soma do todo e não de cada parte em particular.

\section{CONCLUSÃO}

A presente pesquisa aborda discussões importantes a respeito de uma das diversas metodologias que podem ser aplicadas no design de interiores. Neste artigo, se apresenta a ótica utilizada por Fayga Ostrower em seu experimento e os conceitos por ela abordados, tais como elementos visuais e de composição da imagem, evidenciando-se a pertinência em transpô-los para o design de interiores, no intuito de se estabelecerem unidade e equilíbrio do espaço no projeto.

Por meio da análise dos projetos de Patricia Urquiola, nota-se a aplicação prática dos conceitos, e o resultado confere coerência, harmonia e equilíbrio ao ambiente. Cabe ressaltar que a falta de metodologia ou embasamento ao se projetar um espaço, pode resultar no efeito oposto, com sensação de perturbação e falta de equilíbrio visual, por exemplo.

Nesse sentido, percebe-se a contribuição do diálogo entre Arte e Design de Interiores e conclui-se que, apesar de existirem inúmeras formas de se conceber um projeto, os conceitos aqui abordados podem agregar ao repertório e ao resultado final da composição, além de funcionarem como uma diretriz, que auxilia o processo criativo e projetual do designer de interiores ${ }^{11}$.

\footnotetext{
${ }^{11}$ Lina Maria Braga Mendes. Mestre em Linguagem e Educação pela FEUSP; Licenciada em Letras Português e Espanhol pela Universidade Anhembi Morumbi; Bacharel em Filosofia pela FFLCH-USP. E-mail: linamendes@gmail.com.
} 


\section{REFERÊNCIAS}

ARNHEIM, Rudolf. Intuição e Intelecto na Arte. São Paulo: Martins Fontes, 2004

FERRAZ, C. B. O. (2016). Geografia: o olhar e a imagem pictórica. Pro-Posições, 20(3), 29-41. Disponível em:

<https://periodicos.sbu.unicamp.br/ojs/index.php/proposic/article/view/8643386>.

Acesso em: 15 maio 2020.

GIBBS, J. Design de interiores. Guia útil para estudantes e profissionais. São Paulo: Gustavo Gili, 2010. p.70.

GOMES FILHO, João. Gestalt do objeto: sistema de leitura visual da forma. 9. ed. São Paulo: Escrituras Editora, 2009.

GRIMLEY, C. Cor, espaço e estilo. São Paulo: Gustavo Gili, 2017.

OSTROWER, Fayga. Universos da Arte. Campinas, SP: Editora da Unicamp, 2013.

SILVA, Wanessa Cordeiro. Aquarelas: A linguagem das cores em Fayga Ostrower. 2016. 121 f. Dissertação (Mestrado em Teoria e História da Arte) - Universidade Federal do Espírito Santo, Espírito Santo, 2016.

Sites:

ARCHDAILY. Hotel Mandarim Oriental. Disponível em: <www.archdaily.com.br/br/0176664/hotel-mandarin-oriental-barcelona-oab?ad_medium=gallery>. Acesso em: 17 maio 2020.

ARCHILOVERS. Hotel Room Mate Giulia. Disponível em:

<www.archilovers.com/projects/176656/hotel-room-mate-giulia-milan.html\#info>.

Acesso em: 20 maio 2020.

PATRICIA URQUIOLA. Mandarin Oriental Hotel. Disponível em:

<http://www.patriciaurquiola.com/architecture/mandarin-oriental-2>. Acesso em: 22 maio 2020. 